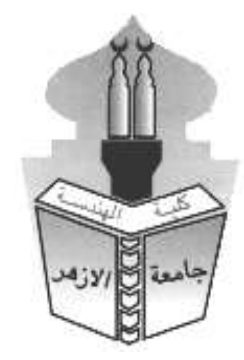

\title{
BIOMEDICAL HANDHELD EMBEDDED SYSTEM
}

\author{
Mohamed A. Soliman ${ }^{1, *}$, Ibrahim F. Tarrad ${ }^{\curlyvee}$, Ayman M. Wahba ${ }^{r}$ \\ ${ }^{1}$ Electrical \& Computer Engineering Department, Higher Technological Institute, $10^{\text {th }}$ of Ramadan City, Egypt. \\ ${ }^{2}$ Electrical Engineering Department. Faculty of Engineering, Al-Azhar University, Cairo, Egypt. \\ ${ }^{3}$ Computer \& Systems Eng. Dept., Faculty of Engineering, Ain Shams University, Cairo, Egypt.
}

*Corresponding Author E-mail: m.ahmedsoliman@gmail.com

\begin{abstract}
In recent times, most hospitals in the world are crowded and facing the problem of increasing numbers of patients, specifically with the spread of infectious viruses such as Covid-19 and other chronic diseases such as cardiac disease and Geriatrics diseases, therefore patients need continuous and accurate emergency care. The heart rhythm disturbances, unsaturated oxygen in the blood, and high-temperature may be a high risk to the life of the patient. Most hospitals and medical quarantines have a shortage of Intensive care units (ICU) rooms. This paper discusses the design and improvement of the Patient monitor device for converting it to a handheld device to be small in size (L145 x W85 x D45 mm), lite Wight (398 gm.), low-cost nearly (98USD), easy-to-use by touch screen, and secure connections to internet with (SSL) protocol[22]. The maximum errors of ECG HR is $1.408 \%$, and SPO2 is $1.075 \%, \mathrm{SPO} 2 \mathrm{HR}$ is $1.408 \%$, temperature is $0.549 \%$, BP systolic is $2.439 \%$, BP Diastolic is $3.448 \%$, The patient can use the device anywhere by himself, in an easy way and safely. This device measures physiological parameters such as (blood pressure by using (MAP) method, the saturation of oxygen in the blood (SPO2) by photoplethysmograph (PPG), the electrical and muscle movement of the heart through 3Lead (ECG), temperature, and finally, Heartbeat rate by different three ways). All data are monitoring on Smart Touch TFT, and storing the Main information of patient for more than thirty days Continuously on SD. Also using (IoT) technology to transfer all patient information in real-time through ESP8266 to Android or Website to follow-up the patient directly and permanently by Doctor or Caretaker, The device gives some important Speech voice sentences and sound alerts to improve the health of the patient. to design and implement the system with low cost, we are using the traditional lowcost sensors, with changing the large bulker analog circuits to intelligent signal conditioning by using (CMRR) with Sallen-Key Active filter and low-cost successive approximation 12-bit ADC MCP3208, with DAC MCP4728 and improving the efficiency by DSP software Specifically FIR and Moving Average Filter. This paper not using any Platforms under (RTOS), but using embedded BASIC language with the wide-range microcontroller PIC18f46k22, to keep the program in small size of memory and low power consumption
\end{abstract}

KEYWORDS: Sensor and Signal Conditioning, PIC18f46K22, ESP8266, MAP, PPG, DSP, Smart Touch TFT. 


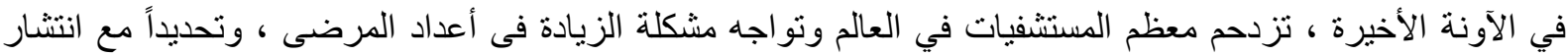

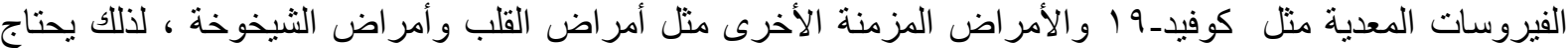

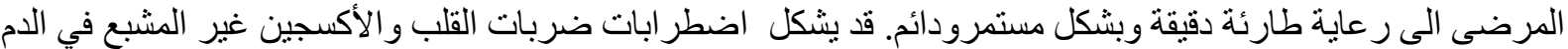

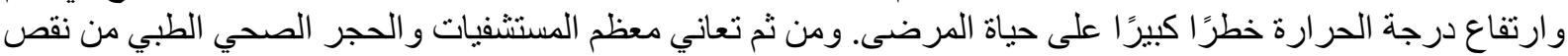

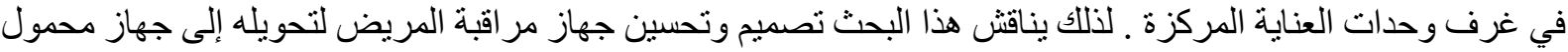

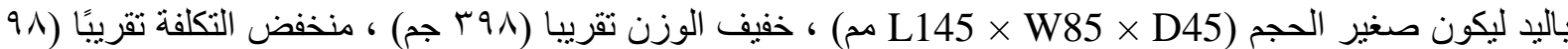
دو لارًا) ، سهل الاستخدام عن طريق شاشنة تعمل باللمس ، و اتصالات آمنة بشبكة الإنترنت باستخدام برونوكول (SSL)

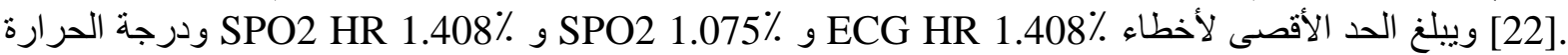

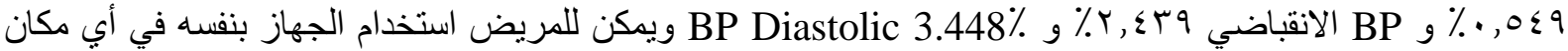
بطريقة سهلة وآمنة. يقيس هذا الجهاز المعلمات الفسيولوجية مثل (ضغط الدم باستخدام طريقة (MAP) ، وتشبع الأكسجين

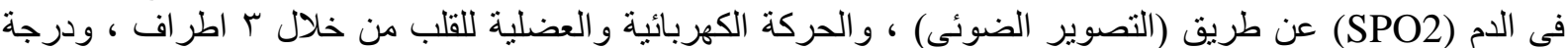

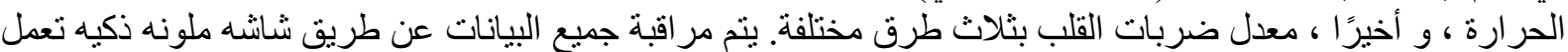

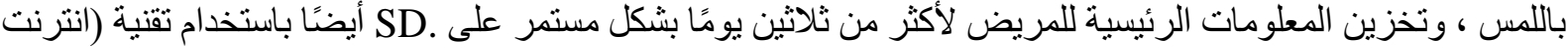

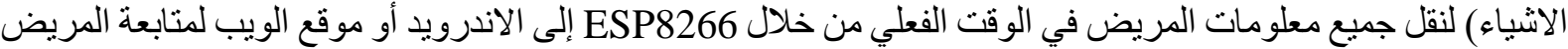

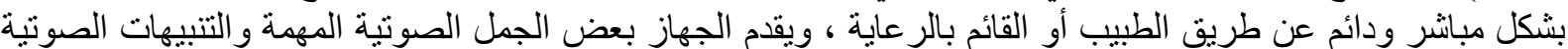

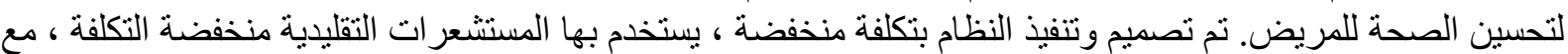

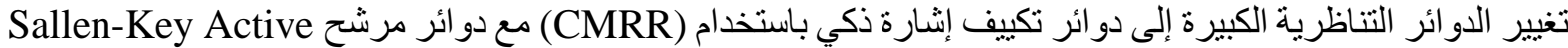

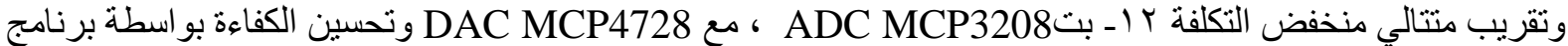

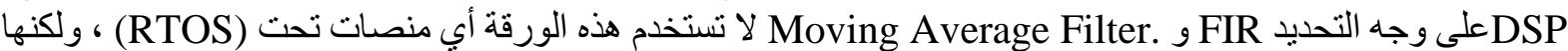

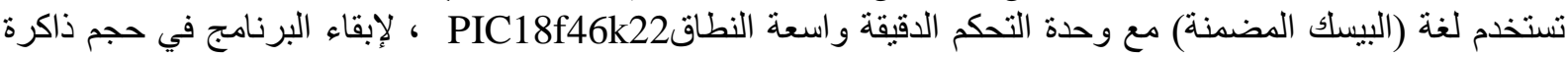
صغير و استهلاك منخفض للطاقة

الكلمات المفتاحية : الحساسات ودوائر التكيف , PSP18f46K22, ESP8266 , أقصى ضغط شرياني , التصوير

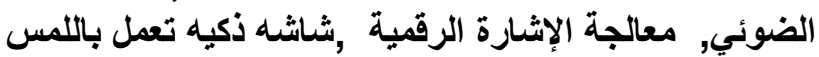

\section{INTRODUCTION}

The World Health Organization reports that 17.9 million people died in 2016, from cardiovascular disease, and accounting for $31 \%$ of the number of deaths this year. and More than $75 \%$ of death. people occur in low and middle-income countries [13], also in recent times, the problem of increasing the number of patients in the hospitals, specifically after the spread of new infectious viruses such as COVID-19, the World Health Organization recommends the necessity of social distancing between the people to decreasing the chance of Disease spreading, also chronic diseases such as cardiac disease and Geriatrics diseases need Permanent health care and emergency. This is difficult to provide health care for these large numbers of infected people, it may be represented Heart rhythm disturbances, unsaturated oxygen in the blood, high temperature, and blood pressure are a great danger to the lives of patients, especially old People.

the growing progress in technology and the fast development for modern digital electronics and communication technologies And the fast-spreading of the technologies make the people using 
it too much all times, such as smartphones, tablets, PDAs and computers with Internet services, electronic healthcare systems have the best opportunity to design new ideas of biomedical devices to upgrading the measurements performance and efficiency of the devices options. Smartphones and personal computers have the facility to connect with the Internet, and the Internet has become available even in poor and far places by the easy way and low cost. so it is useful to use smartphones and computers to transfer physiological parameters from Patient to doctor or caretaker, this gives the doctor chance to save the time needed to diagnoses and check for disease history and make a diagnosis in a faster and more accurate. The biomedical handheld system enables doctors to monitor their patients anywhere without needing the patient to stay in hospital so that medical services become fast.

Burhanuddin et al [1], focused on hardware circuit design for patient healthy monitor to improvement the performance and measuring accuracy, and most of researchers using a digital sensors modules [3-5], its calibrated for reading but not confortable to attached with human body and also not available in all markets, some researchers prefer to using the microcontroller platform or emulators kits for processing and data analyses[4,5], this platforms have many unused peripherals circuit then the power consumption and cost is effected, wireless sensor network (WSN) integrated with the internet by IoT, its a good solution for medical data traveling or telecommunication [3,5].

The point of paper, design and implement the complete good performance prototype of a biomedical handheld embedded system hardware and software, It does the same functions of (ICU) patient monitor device but portable, using IoT technology, and using reliable medical classified sensors Which characterized by high-quality specifications, comfortable, and easy to connect with the body, also cost-effective and widely used in the market. The challenges are how to use a wide-Range microcontrollers PIC18F46K22 with medium performance and Lowcost, to design and implement the portable biomedical system, with high accuracy and solve the not availability of Digital Signal Controller DSC hardware in the wide-Range 8-bit microcontroller [14], by using software FIR filter and Moving Average Filter working with $\mathrm{DAC}$ and $\mathrm{ADC}$ to sure the signal always within the data analysis range and increasing the performance of filters, store the vital data inside SD card more than 30 days continuously, and send the vital data by a new technology of IoT to smartphones and computers through Cloud servers[5].

The next section provides discusses system design, Section 3 Biomedical Data Acquisition, Section 4, Processing and Peripherals, Section 5, Software program design, Section 6, Results and testing, Section 7, conclusion, finally, future work.

\section{SYSTEM DESIGN}

The general design architecture of the Biomedical Handheld Embedded system is classified into two main units, Biomedical Data acquisition, processing, and peripherals (see Fig. 1). 


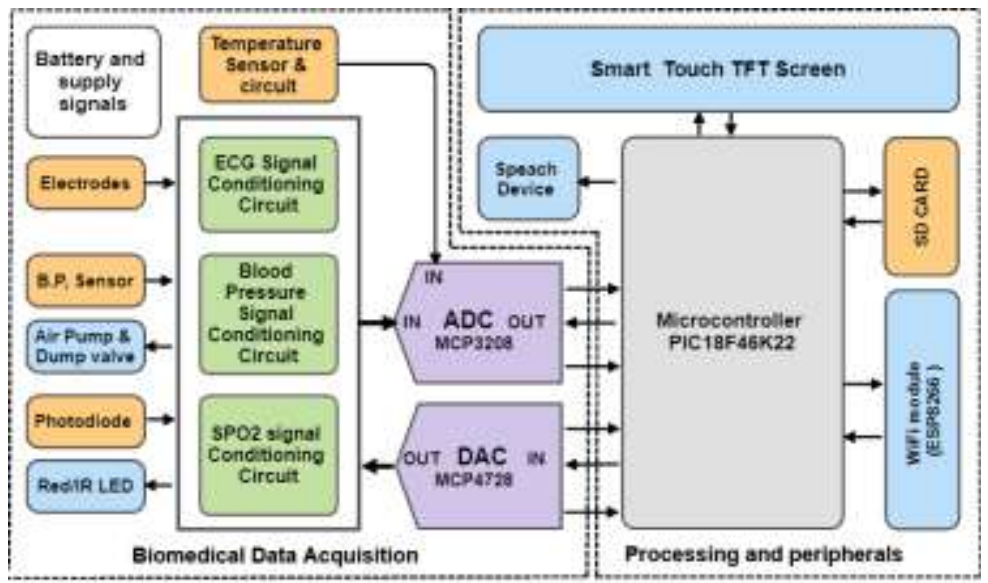

Fig. 1: Biomedical Handheld Embedded system

\subsection{Biomedical Data Acquisition}

The non-invasive medical sensors attached to the patient body, work to sensing the physiological or vital parameters, sensors should be connected with a signal conditioning circuit, to convert the physiological parameters to small electrical signals by using the amplification, filtering, and Digital Signal Processing, to modify the signals to be easy for reading, and compatible with processing unit, The ADC and DAC circuit work together as close loop system to detect the error input signal[15] such as ( DC component or signal outside of range) and correct it to get an accurate result, the vital parameter is Temperature, ECG signal, Blood pressure signal, and Oxygen saturated (SPO2) signal..

\subsection{Processing and Peripherals}

The Biomedical data acquisition unit sending the modified signals to the microcontroller to execute the signal processing calculations, which connected with important peripherals such as Smart TFT display to monitoring and notification the physiological parameter of the patient, SD memory card used to permanently storage all vital signals calculated from microcontroller for at least 30 days, to show the doctor this data later for make good diagnoses and give the patient a suitable treatment, a speech voice notification peripheral used to tell the patient some warning messages about his health and connection troubleshooting of the handheld device, finally Wi-Fi module for IoT communication between the patient and doctor or caretaker.

\section{BIOMEDICAL DATA ACQUISITION}

\subsection{Temperature signal}

A Negative Temperature Coefficient (NTC), is a sensor preferred to selected in this design because it's the most common sensor using in most medical equipment, and its characterized by low cost, fast reading response, good stability over time, and available in all medical market, but it has two bad factors, Self Heating resistance tolerance, and the non-linear characteristics or resistance ratio slop. The Self Heating gives a very small error for the Human body temperature range, and the nonlinearity Can be fixing this problem by using many Theories, one of them is called a Simple Theory or BETA Value method [16] (see Equation 1),

$R=A e^{(\beta / T)}$ 
This calculated in the software design. The human body temperature range is between $35^{\circ} \mathrm{C}$ to $41^{\circ} \mathrm{C}$, then the relative BETA calculated from $25-50^{\circ} \mathrm{C}$ to select a narrow operation range to solve the nonlinear resistance slop to increasing the accuracy as possible. The average error $= \pm 0.026$ to make the difference between NTC Typical output Curve and BETA Calculated Curve is very small (see Fig. 2), the NTC thermistor does not need a complex signal conditioning circuit, just voltage divider circuit enough get signal from a sensor, the $2.2 \mathrm{k}$ NTC is connected as pull-down resistor to get voltage increment with direct proportionally with temperature increment..

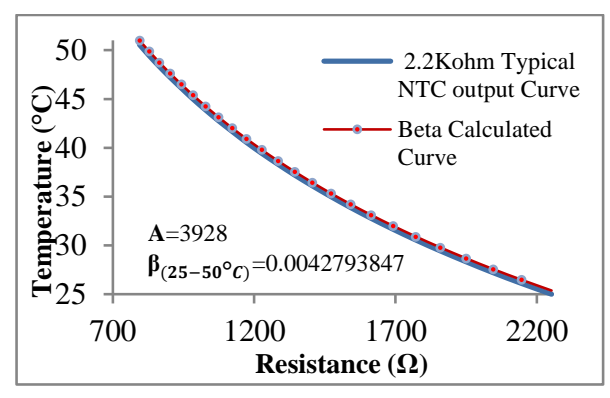

Fig. 2: NTC Typical output with Beta Curve

\subsection{ECG signal and circuit}

The Electrocardiogram (ECG) sensor electrode detects the small human body signals and converts them from a physiological signal to an electrical signal with voltage amplitude from $1 \mathrm{mV}$ to $5 \mathrm{mV}$. The ECG circuit has 3Lead electrodes (RA, RL, and LA) attached to the body chest [6], Many signals are emitted from the body, such as Muscle, Nerve, and Brain signals (EMG, ENG, and EEG), All of these signals can be obtained from anywhere in the body, but the specified Location (see in Fig. 3) make the ECG signal highest as possible, and other unwanted signals will be wreaked.
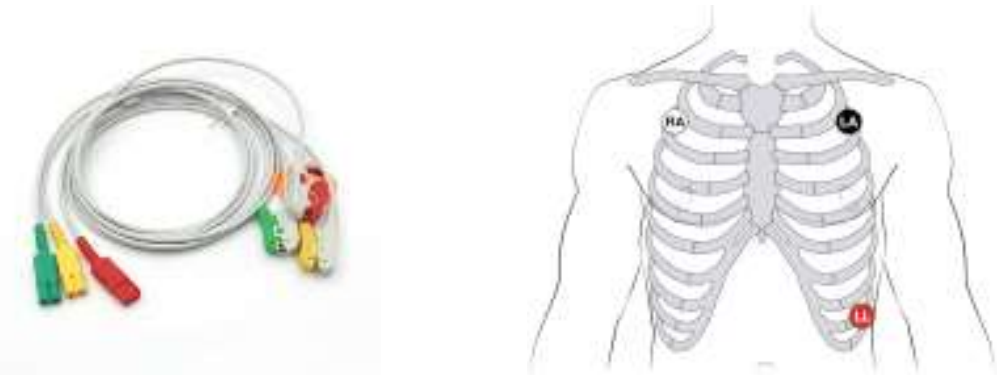

Fig. 3: ECG Electrode on the Body Location

The ECG circuit schematic diagram (see Fig. 4). It begins with an Instrumentation Amplifier (I.A.) with Gain $=7$, used to amplify a small analog signal generated from the ECG sensor with good isolation by Common Mode Rejection Ratio (CMRR)[1,2] . 


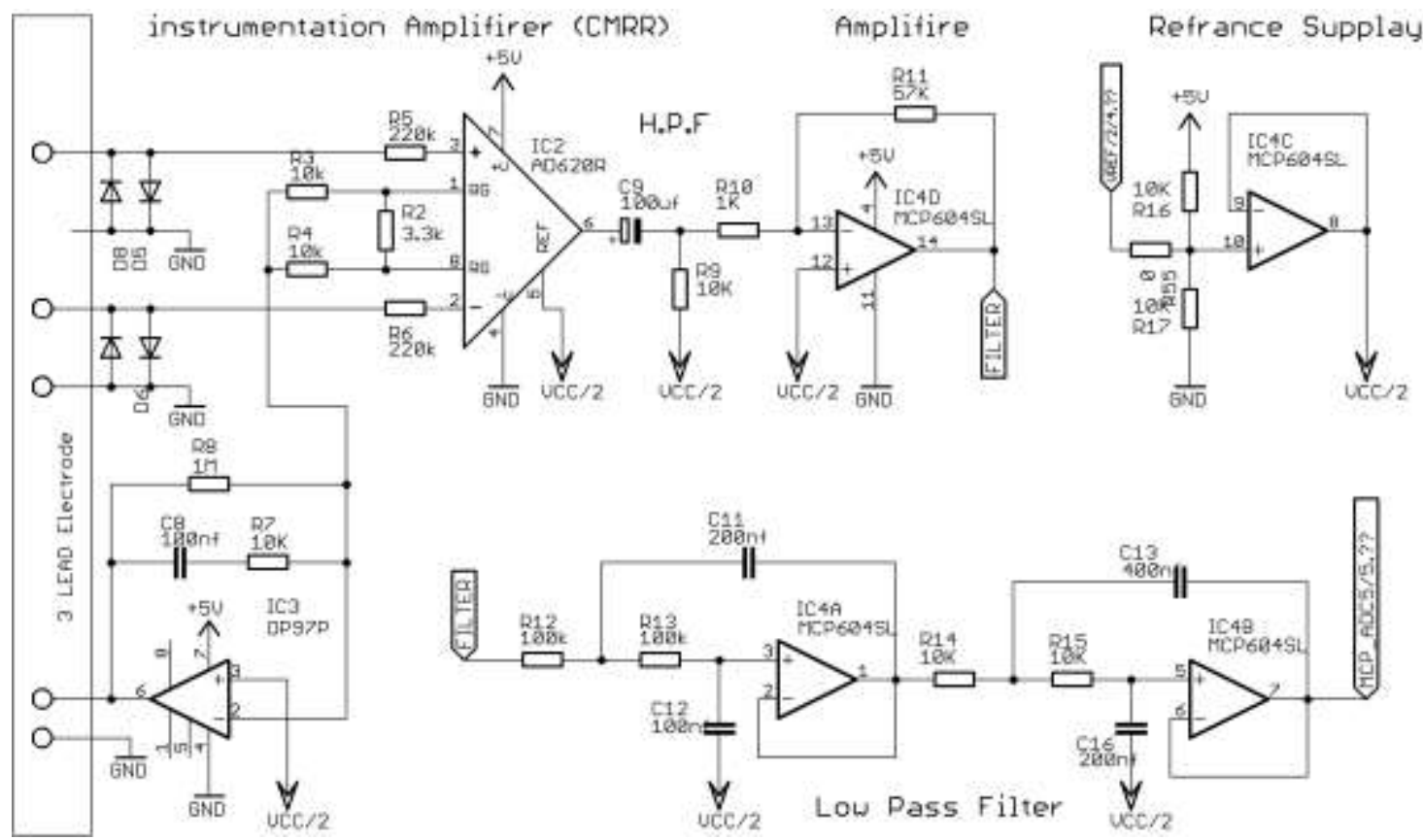

Fig. 4: ECG signal conditioning circuit diagram

the analog output signal from I.A. is filtered by using High pass filter with Cutoff frequency $=0.05 \mathrm{~Hz}$, the function of the filter removes the Low-frequency components and $0.05 \mathrm{HZ}$ lower frequency consist of ECG signal standard reading from adult and children, the second function of High pass filter to remove the DC Components can appear from the Instrumentation amplifier.

After remove the dc components from the signal will be amplification with Gain= 57 from inverting OP-Amp, this OP-Amp inverting input pin, connecting with DAC (VREF),

The next stage of the signal conditioning is a 4th order Butterworth Sallen-Key Low Pass Filter [17], this filter is designed with cutoff frequency $=150 \mathrm{~Hz}$, it will be suitable for adult only, the 4th order good for cost and signal quality (see Fig. 5.b),

The output signal from Low Pass Filters is ready to read from the PIC18F46K22 microcontroller via 12bit ADC MCP3208, to complete the signal analyses and signal calculations.

The reference supply signal of the ADC and all OP-Amps will be connected with DAC MCP4728 after protected the signal by unity feedback voltage follower amplifier to change the reference voltage (VREF) for all signals conditioning to moving the ECG signal up/ Down by software program to ensure the signal found inside the correct range and did not have any clapping or distortion wave shaping. 


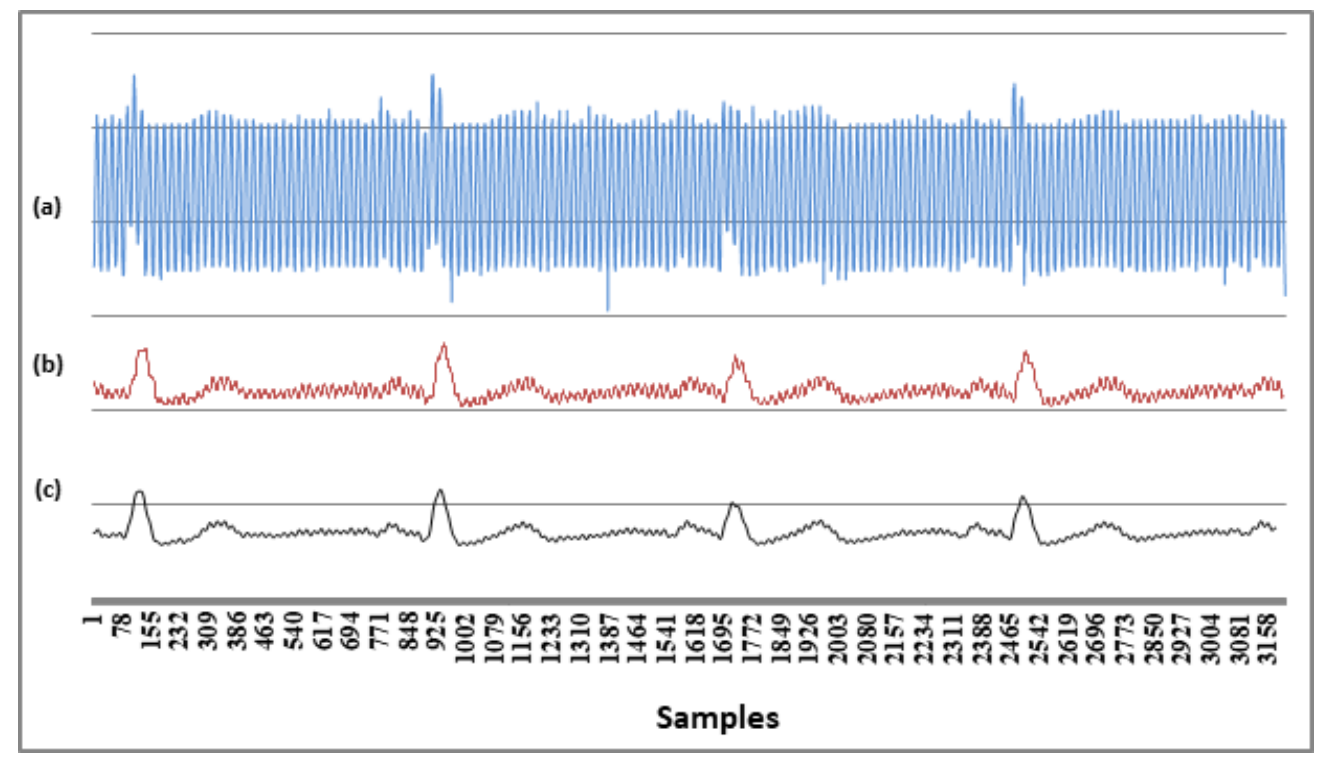

(a) Signal after IA. (b) Signal After FIR LPF

(c) Signal after applied Moving Average Filter

Fig. 5: ECG Signal before and after Sallen-Key effect

A Moving Average Filter (MA Filter) is a simple software Low Pass FIR, with Finite Impulse Response, the filter used for smoothing the signal and its prefer to using it in the final stage, the smoothing quality of the output signal dependent on the length of the filter [7], the ECG filter length $=10$ samples of input and calculates the average of those 10 samples by (equation 2) to produces a single output point. MA Filter structure using to filter unwanted noisy components from the intended signal it is means the filter has an excellent time-domain response (see Fig. 5.c)

$$
y[n]=\frac{1}{L} \sum_{k=0}^{L-1} x[n-k]
$$

\subsection{Blood Pressure signal and circuit}

A Blood Pressure circuit starting with high-reliability MEMS pressure sensor MPS20N004 the sensor working with a pressure range from 0.5 to 8 psi with linearity $=0.3 \%$ in full scale, connected with Instrumentation Amplifier designed by using two amplifiers is more suitable and cost-effective, the function of I.A. to be amplifier the analog signal acquired from the pressure sensor Wheatstone bridge, with Gain $=64$ The output signal distribute to two directions. First direction to ADC2 to representing the cuff pressure amplitude, and the second direction to a high-pass filter with cutoff frequencies of $0.16 \mathrm{~Hz}$ to remove any DC component, the signal amplified with Gain $=50$ by a non-inverting amplifier.

Low Pass Filter is formed by 2nd order Butterworth Sallen-Key Filter it designs by cutoff frequency = $150 \mathrm{~Hz}$, The output from the Low Pass Filter, representing the oscillation heartbeat signal, The filtered signal sent to the microcontroller in processing and peripherals unit via the ADC3 channel of MCP3208, to complete the processing and digital signal analyses.

Air pump and dump valve signals will be isolated by optical-isolator PC817 controlled directly from the microcontroller signal. 


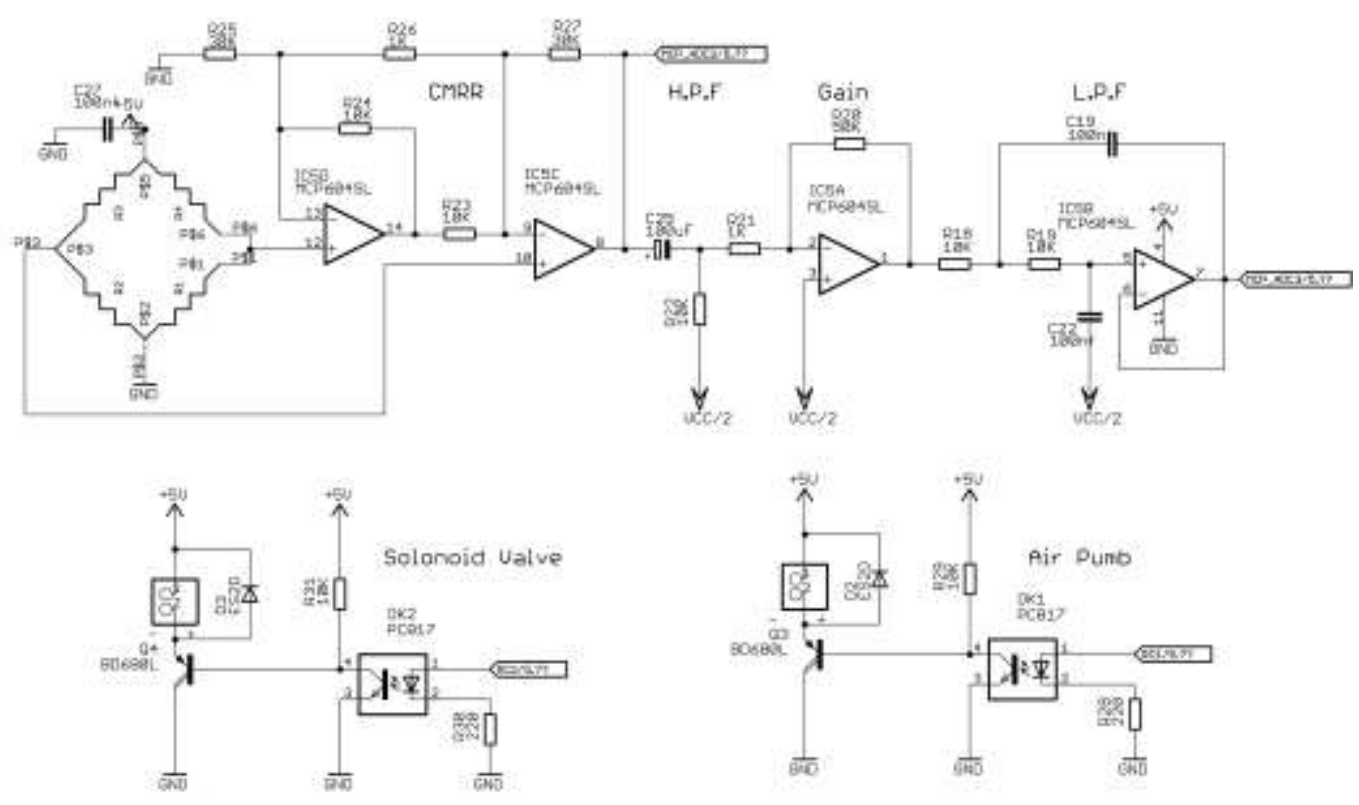

Fig. 6: Blood Pressure signal conditioning circuit diagram

Blood pressure oscillometric method dependent on the pressure amplitude pulsations taken during measurements, by placing the cuff on the left arm for the patient and is connected to an air pump, pressure sensor, and turn on the air Pump. Cuff is inflated to be greater than the highest systolic value can be reached, then the Dump valve is a leakage the air slowly and the cuff is deflated. When getting the systolic pressure, the heartbeat pulses begin to appear. This signal is used to get the heartbeat rate by calculation. The Pulses increasing in amplitude to become a Maximum value of pressure it has called Main Arterial Pressure (MAP), and then decreases the pressure to be very small. (See Fig. 7) will show the measuring cuff pressure with heartbeat pulsations from the biomedical handheld device, by using the ISDS205x digital oscilloscope for plotting.

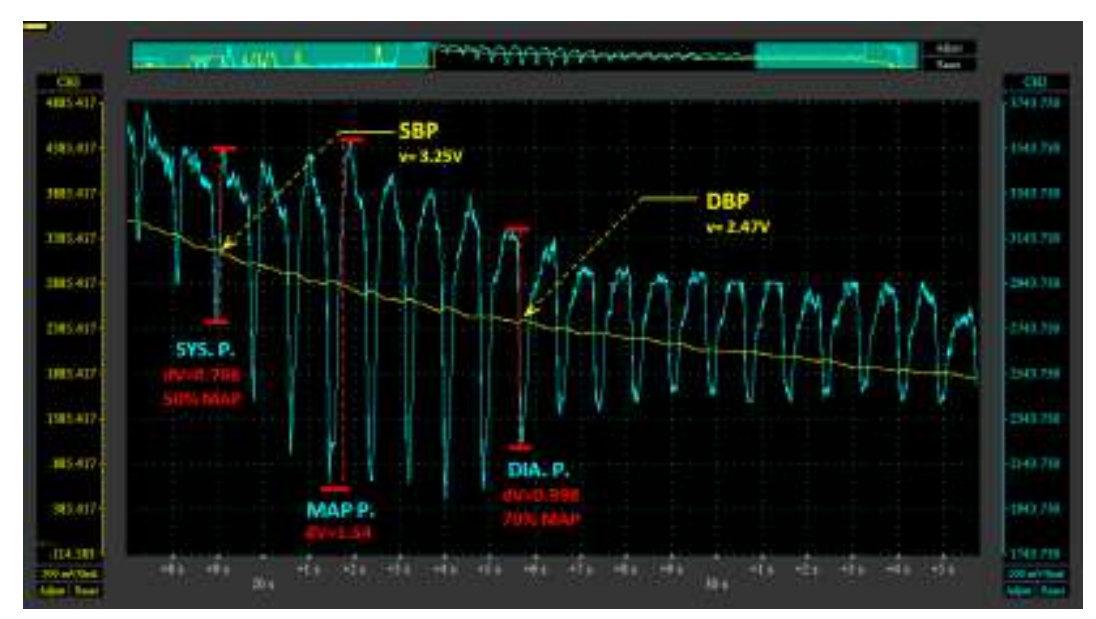

Fig. 7: The measuring method cuff pressure with heartbeat pulsations 


\section{BIOMEDICAL HANDHELD EMBEDDED SYSTEM}

The biomedical handheld device recovers and calculating the MAP, by latching the largest pressure amplitude that appears from cuff pressure.

Systolic and Diastolic values are calculated by considering that Systolic pressure is approximately equal to the pressure measurement when a pulse reached $70 \%$ from the MAP pulse latched when the pressure is more than MAP value.

Diastolic pressure is equal to the cuff pressure value reading from the buffer when pulse amplitude within 50\% from the MAP amplitude latched when the cuff pressure is lower than MAP value [8, 18]. Table1, explains the calculation of Systolic and Diastolic for three different Patient tested by the biomedical handheld device.

Table 1. Measuring Sys./ Dia. for three different patient

\begin{tabular}{|c|c|c|c|c|c|}
\hline \multicolumn{2}{|c|}{ Patient (A) } & \multicolumn{2}{|c|}{ Patient (B) } & \multicolumn{2}{|c|}{ Patient (C) } \\
\hline $\mathrm{mmhg}$ & ADC 3 & $\mathrm{mmhg}$ & ADC 3 & mmhg & ADC 3 \\
\hline 142 & 778 & 168 & 518 & 169 & 197 \\
\hline 133 & 794 & 155 & 544 & 165 & 205 \\
\hline 126 & 869 & 145 & 696 & 152 & 270 \\
\hline 120 & 1163 & 136 & 768 & 142 & 317 \\
\hline 114 & 1450 & 128 & 1008 & 133 & 350 \\
\hline 107 & 1599 & 121 & 1284 & 125 & 422 \\
\hline 103 & 1605 & 114 & 1340 & 118 & 424 \\
\hline 98 & 1691 & 109 & 1514 & 112 & 639 \\
\hline 94 & 1570 & 103 & 1477 & 106 & 900 \\
\hline 90 & 1426 & 98 & 1396 & 100 & 991 \\
\hline 87 & 1365 & 93 & 1226 & 95 & 1105 \\
\hline 83 & 1170 & 89 & 1203 & 91 & 1201 \\
\hline 80 & 1049 & 84 & 949 & 87 & 1029 \\
\hline 78 & 864 & 80 & 653 & 82 & 1104 \\
\hline 74 & 840 & 77 & 438 & 79 & 917 \\
\hline 71 & 792 & 73 & 418 & 75 & 642 \\
\hline 69 & 586 & 70 & 296 & 72 & 586 \\
\hline \multicolumn{2}{|c|}{ MAP $=98$} & \multicolumn{2}{|c|}{ MAP $=109$} & \multicolumn{2}{|c|}{ MAP $=91$} \\
\hline \multicolumn{2}{|c|}{ SYS. $=124$} & \multicolumn{2}{|c|}{ SYS. $=137$} & \multicolumn{2}{|c|}{ SYS. $=117$} \\
\hline \multicolumn{2}{|c|}{ DIA. $=83$} & \multicolumn{2}{|c|}{ DIA. $=86$} & \multicolumn{2}{|c|}{ DIA. $=78$} \\
\hline
\end{tabular}

\subsection{Pulse Oximeter Design}


A Pulse Oximeter calculates the oxygen saturation ratio (SpO2) of human blood by photoplethysmograph (PPG) method by using two different light wavelengths, red light about $660 \mathrm{~nm}$ and infrared light about $940 \mathrm{~nm}$ [15].

Oxy-hemoglobin ( $\mathrm{HbO} 2)$ absorbs a lot of infrared light and allows the red light to pass, and Deoxyhemoglobin $(\mathrm{Hb})$ absorbs a lot of red light and allows the infrared light to pass, (see Fig. 8.a), The microcontroller flashes the RED/IR lights turned on one by one or alternative from the finger to a photodiode detector.

The photodiode detects the non-absorbed light from RED/IR LED to calculate the absorbed light come from the finger, the signal conditioning circuit will convert an absorbent light to a voltage signal; the amplitudes of the RED/IR signals are measured and converted to Rout mean square value (Vrms) to produce a Ratio as given by Equation 3 .

The $\mathrm{SpO} 2$ is determined by using the Ratio value at a look-up Table 1, that is made up of empirical formulas. (See Fig. 8.b).

$$
\text { Ratio }=\frac{\operatorname{Red} A C_{V r m s} / \text { Red } D C}{I R A C_{V r m s} / I R D C}
$$

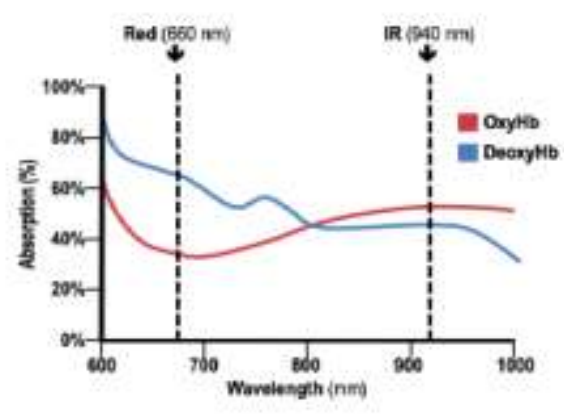

(a) Hemoglobin light absorption graph.

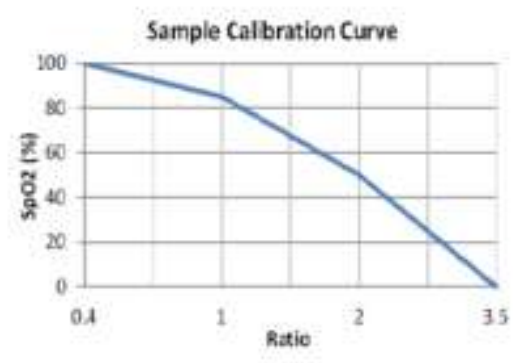

(b) Sample Calibration Curve[14]

Fig. 8: SPO2 absorption and calibration curve

A DUAL SPDT analog switch (LN3005) driven by two PWM signals from the microcontrollers turns the red and infrared LEDs on and off alternately, the intensity of the light is changing by DAC2 and DAC3 (MCP7428) that depending on the finger skin tissue thickness and skin color.

The non-absorbent light signal will be received from a finger it will be detected by photodiode from transimpedance signal conditioning circuit to convert the photo intensity from microampere by the photodiode and convert it to millivolt.

High Pass Filter filters the signal with $59 \mathrm{~Hz}$ cutoff frequency, which is designed to reduce the background light interference.

The output of the High pass filter is sent to a second non-inverting amplifier with a Gain=22 to magnifier the signal.

ADC6 and DAC1 work together by software commands to set the signal in the probably placed the output signal level in reading range from the microcontroller, by change the DC offset for VREF. 


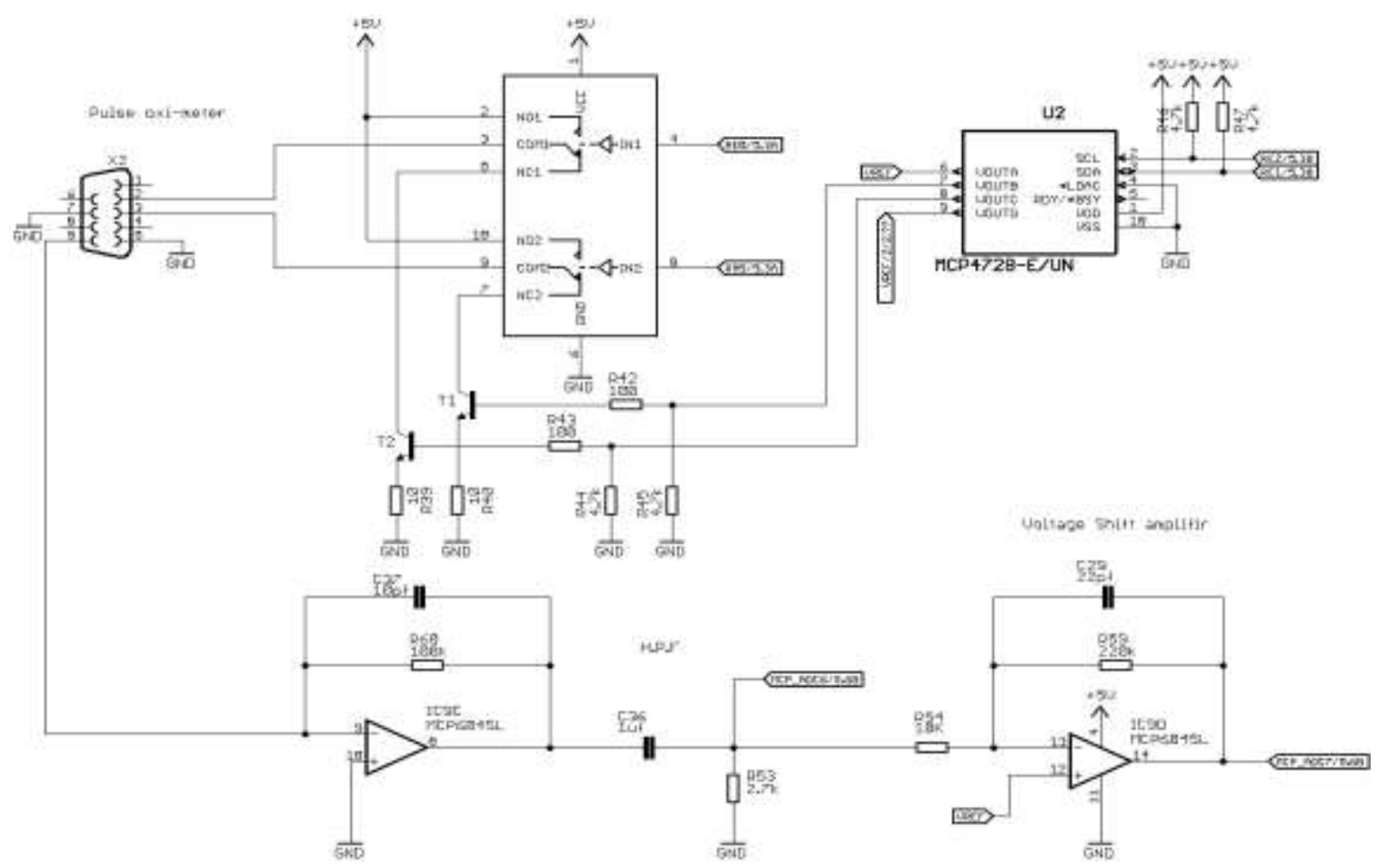

Fig. 9: Pulse Oximeter signal conditioning circuit diagram signal conditioning circuit diagram

The output signal from the analog signal conditioning circuit is connected to the ADC7, the sample captured during RED/IR LED's at On-time period, and one sample captured during LED's at OFF-time period to neglect the Unwanted light in the dark period [19]. PIC18F46K22 applying the FIR Filter[9] (Equation 4).

$$
y[n]=x[n] * a 0+x[n-1]^{*} a 1+x[n-2] * a 2+\ldots+x[n-N+1] * a N-1
$$

Digital filter designed by ScopeFIR and Microchip's Digital Filter Design Tool. The FIR Low pass Filter Specifications is

- Sampling Frequency : $200 \mathrm{~Hz}$

- Passband Frequency : $99 \mathrm{~Hz}$

- FIR Window: Hamming

- Passband Ripple :-0.1dB

- Stopband Ripple : $-40 \mathrm{~dB}$

- Filter Length: 64 


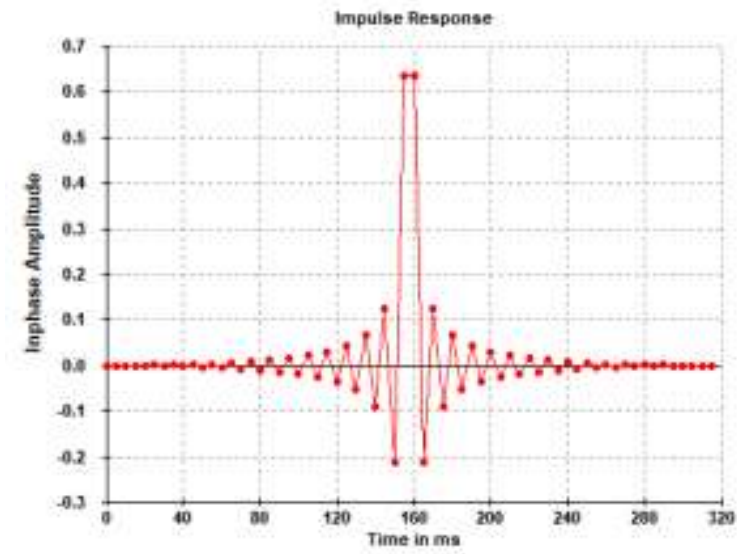

0xFFEA, 0x0017, 0xFFE7, 0x001C, 0xFFDF, 0x0027, 0xFFD2, 0x0036, 0xFFCO, 0x004A, 0xFFAA, 0x0062, 0xFF90, 0x007D, 0xFF74, 0x009B, 0xFF56, 0x00B9, 0xFF38, 0x00D7, 0xFF1B, 0x00F3, 0xFF00, 0x010D, 0xFEE7, 0x0123, OxFED4, 0x0135, 0xFEC5, 0x0141, 0xFEBB, 0x0147, 0x7EB8, 0x0147, 0xFEBB, 0x0141, OxFEC5, 0x0135, 0xFED4, 0x0123, 0xFEE7, 0x010D, 0xFF00, 0x00F3, 0xFF1B, 0x00D7, 0xFF38, 0x00B9, 0xFF56, 0x009B, 0xFF74, 0x007D, 0xFF90, 0x0062, 0xFFAA, 0x004A, OxFFCO, 0x0036, 0xFFD2, 0x0027, OxFFDF, 0x001C, 0xFFE7, 0x0017, 0xFFEA,

Fig. 10: FIR Filter Design and Coefficient parameters

the infrared/red output signal wave shape after applying the FIR filter it's good enough to get the SPO2 ratio ( see Fig. 11.b), but to increasing the accuracy and improvement the infrared signal quality, adding the Moving Average Filter with $\mathrm{L}=50$, the signal will be more smoothing and the SPO2 calculations are accurate (see Fig. 11.c).

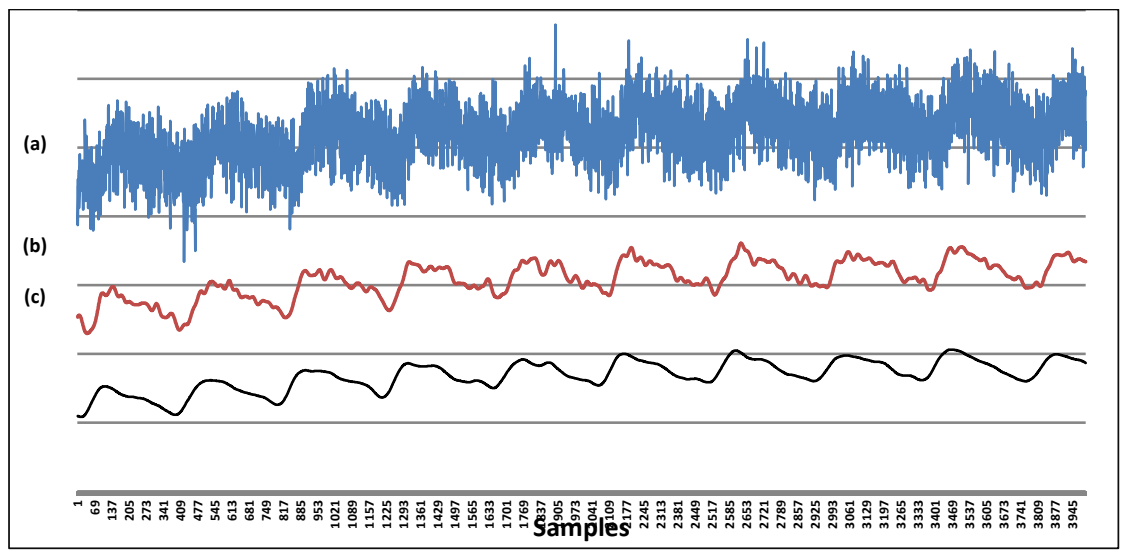

$\begin{array}{ll}\text { (a) Original Signal from ADC6. } & \text { (b) FIR Filtering signal }\end{array}$

(c) Signal after applied Moving average Filter

Fig. 11: Signal Effect by FIR and Moving Average Filter

\section{PROCESSING AND PREIPHERALS}

Used a wide-Range Microcontroller PIC18F46K22 run with the Maximum speed of $64 \mathrm{MHz}$ by using the 4xPLL function Applied to the internal crystal oscillator, the source code is $95 \%$ of flash memory size, and $100 \%$ from internal EEPROM, the package used for a microcontroller and all components are QFP (see Fig. 12).

Special functions running on the microcontroller

- Watch Dog Timer $\quad: 10 \mathrm{~ms}$

- Brown-Out-Reset $: 2.2 \mathrm{~V}$

- Sleep mode $\quad$ : after 1 min of (NO operations) 
- Interrupts

: $5 \mathrm{~ms}$ (200 sample per time)

Signals and ports connection with Microcontroller:

- $\quad$ Digital I/O

- PWM

- USART

- S.W. USART

- SPI

- $\mathrm{I} 2 \mathrm{C}$
: B.P Air Pump, Dump Valve

: RED/IR Led

: Smart touch TFT, ESP8266

: DFplay Voice module

: SD Card, ADC MCP3208

: 12 bit DAC MCP4728

(See Fig. 11) Microcontroller and peripherals circuit diagram

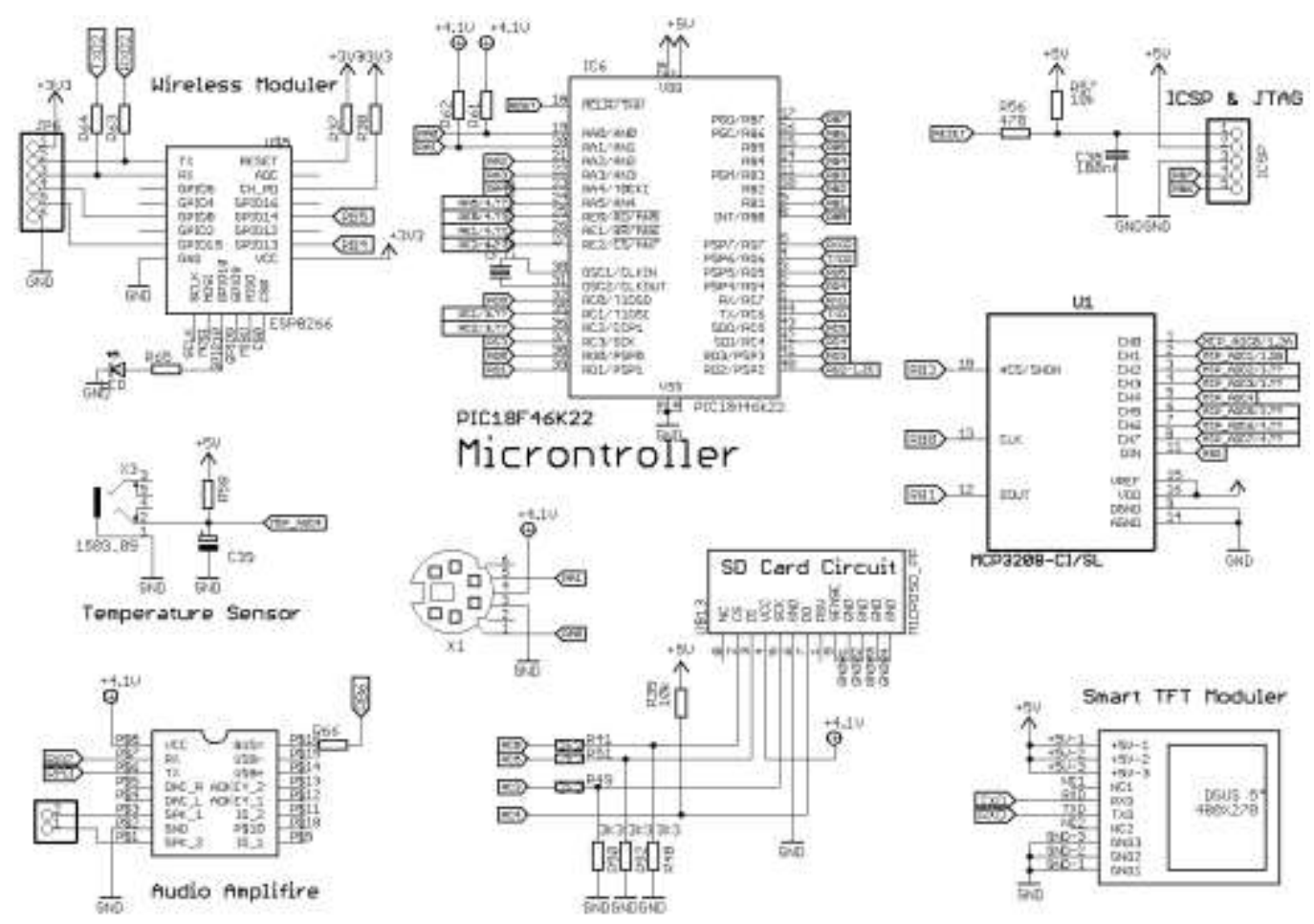

Fig. 11: Microcontroller and peripherals circuit diagram

\subsection{Smart TFT Display}

the smart DMT80480 display resolution is $800 x 480$ pixel[21], clear for small details, the capacitive touch-screen interface decreasing the hardware input buttons, user can be navigated the program to change the setting or reconfiguration some parameters by easy way. The main microcontroller just sends the command or data frame with Baud Rate 345600 bps via USART COM. Port.

\subsection{ESP8266 Communication}

by using the advanced model of ESP8266, which has the Tensilica Xtensa LX106 core, the LX106 Bootloader code is also an open-source code that can be programmed by Arduino application[20,22]. Connected with the main microcontroller by USART COM. Port2.

\subsection{SD Card}

Support up to 64Mb interfacing with PIC18F46K22 by Serial Peripheral interface (SPI) Port, using it to store measured data from (ECG signal, BP signals, SPO2, and Temperature), with 3.3 supply voltage. 


\subsection{Speech voice notification}

Mini DFplay hardware of MP3 player module is good enough to use it with software USART interfacing protocol, to play storing warning massages and troubleshooting attention to increasing the reality of the system.

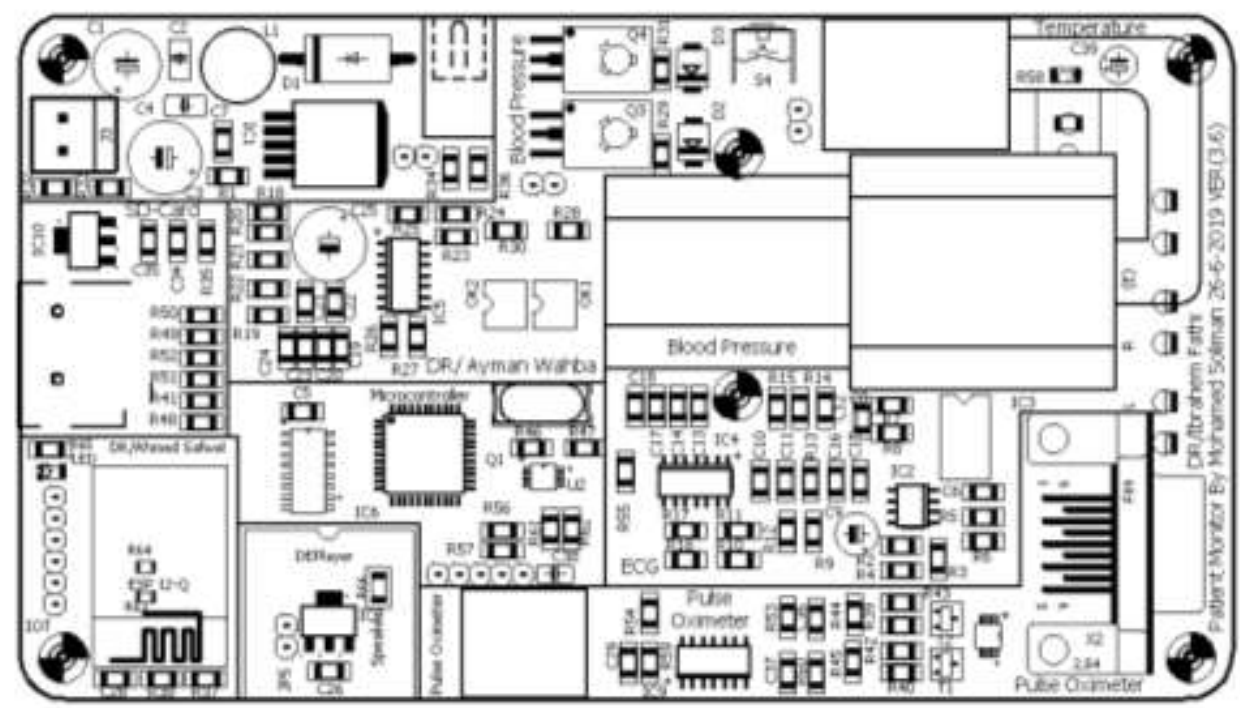

Fig. 12: Top layer view Circuit Layout

\section{Software Program Design}

Five main parts of software programs available in the system design (Main Embedded microcontroller PIC18F46K22 program and interrupt routine, the standalone ESP8266 program, the smartphone medical program, and Smart TFT Touch screen program).

\subsection{Main program and interrupt routine}

The main embedded microcontroller program is divided into two parts (main program and interrupt services). The main program is started by initializing register parameters and I/O ports, such as DAC, ADC, MP3 DFplayer, Red/IR SPO2 signals, and SD card, and initials the USART COM. port for the smart TFT Touch screen with BaudRate 345600 bps, and 9600 bps for ESP8266 COM. Port. Read the stored FIR Coefficient parameters from internal EEPROM and some other configuration and setting values such as final measured values stored.

start the screen and check all hardware connection, now configure the Timer 0 to work in an interrupt mode with a period $=5 \mathrm{~ms}$ or 200SPS to get samples from all input signal, if not found any interrupt data the microcontroller will be active in the standby mode to get the low hardware power consumption. All configuration commands of screen executed in the main program, it has more than 60 Kbyte of flash memory code, most of them for interrupt service code, when an interrupt happens, and the routines execute this sequence (see Fig. 13.a) 


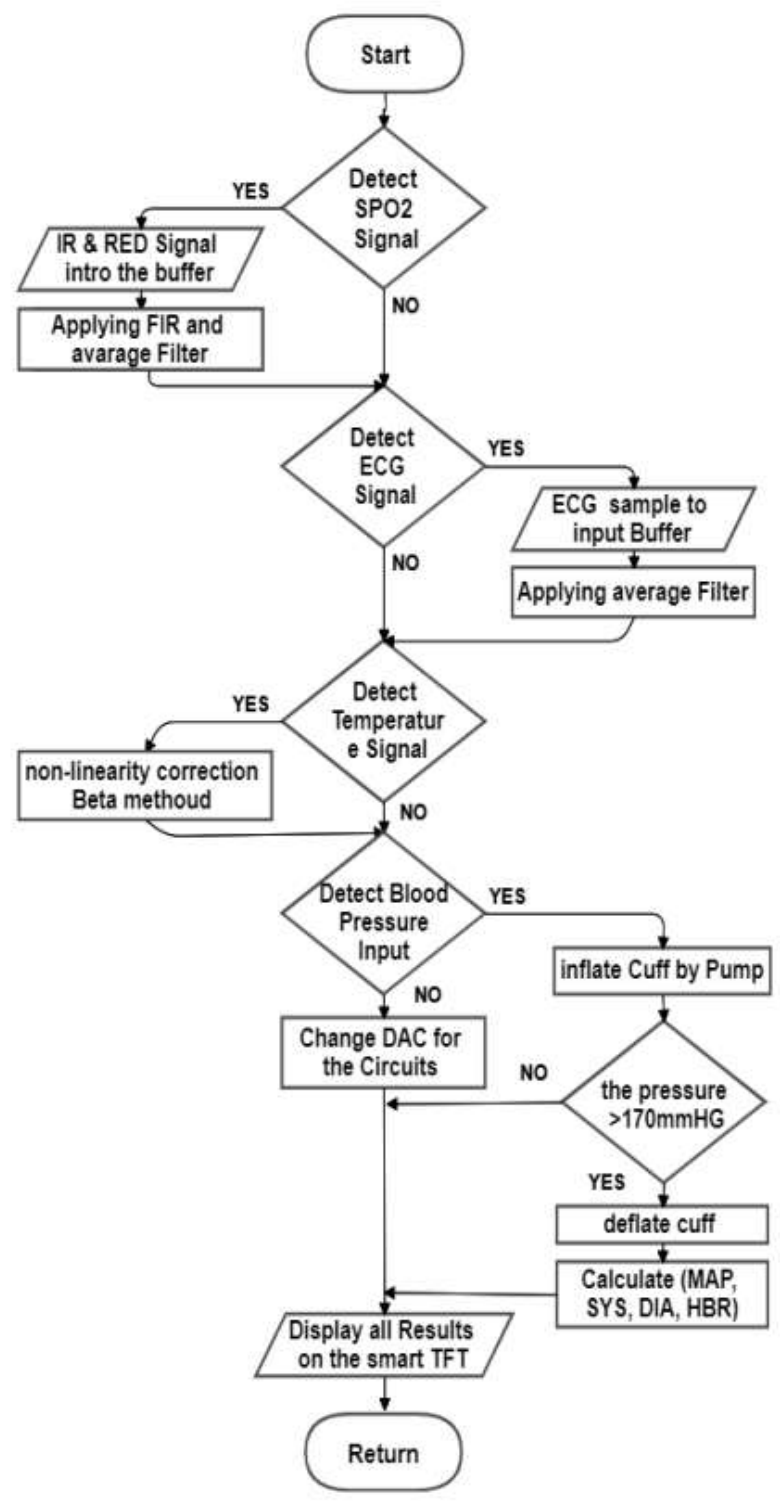

(a) The main program Interrupt Routine

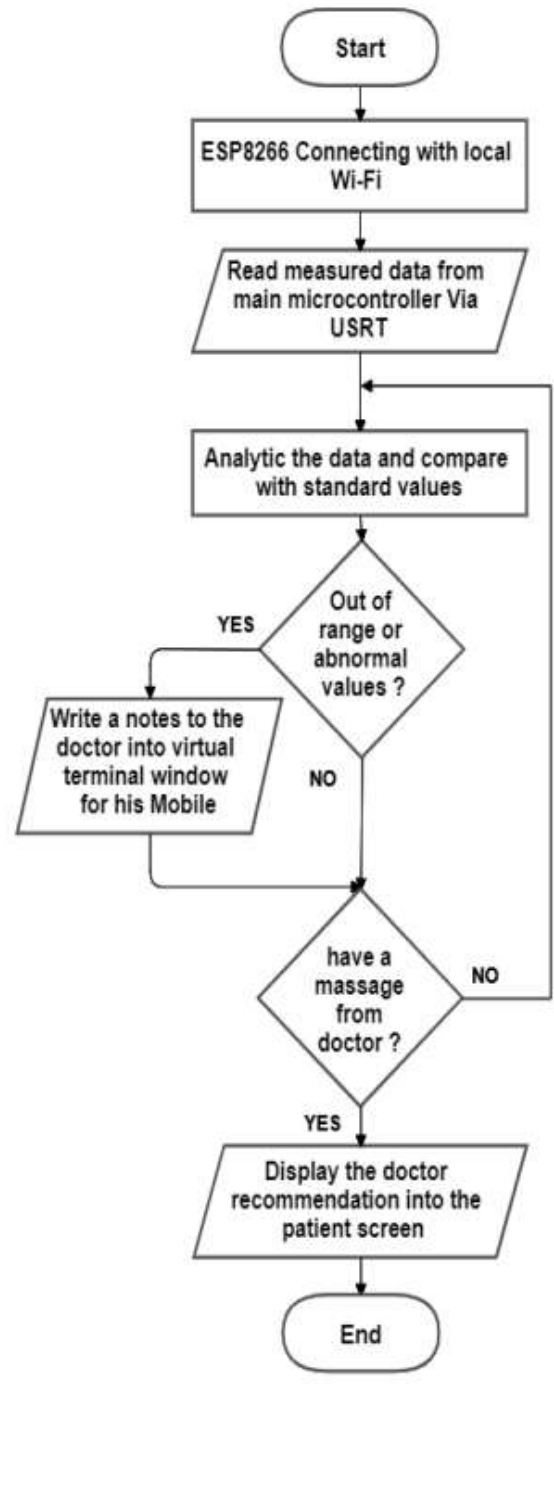

(b) The ESP8266 Flowchart

Fig. 13: Software Flowcharts design idea

- Detect the RED/IR signal From Pulse Oximeter circuit and fill the SPO2 buffer with new samples, then apply the FIR filter and Moving Average Filter to improve the signal wave shape, recover the min/max values of the signal to calculate the SPO2 Ratio and heartbeats rate

- Detect the ECG signal From the ECG circuit and but the reading values of the sample on the ECG buffer to apply Moving Average Filter to improve the signal wave shape, calculate extremely values and capture it to timer1 to calculate the ECG heartbeat rate and compare it with SPO2 heart rate

- Detect a temperature value from ADC and applying the BETA calculation method the solve the nonlinearity and get the real value of temperature with one digit fraction accuracy.

- Detect the Blood Pressure input Button to inflate the cuff and detect the maximum value of pressure, supposed $180 \mathrm{mmHg}$, after that make deflate the pump to out the airflow by $10 \mathrm{mmHg} / \mathrm{sec}$, and sending all values to the Blood pressure buffer to calculate the Maximum Amplitude Pressure MAP, Systolic/ Diastolic values and HBR 
- After calculating all signal and detect All/Any of them out of the range, will reconfigure the DAC to get all of them inside the correct range

- Sending the new measuring values to the Smart TFT display for updating the screen.

\subsection{Smart TFT touch screen programming}

The smart Touch TFT Display is called DGUS[21] graphics application service software, is innovative intelligent GUI software, to quickly develop the high-reliability full-graphics, with the development of simple code, have a flexible operating system without operating speed change, very strong The reliability and stability characteristics, with Baud Rate selected 345600 bps via USART COM. Port to speed up the connection between main embedded microcontroller PIC18F46K22 and Smart TFT with K600 processor, the touch screen module is included with screen circuit design and reading it from USART frame Code. (See Fig. 14). showing the measured vital data, the ECG signal Graph with ECG Heart rate, $\mathrm{SPO} 2$ signal graph, and the percentage value with SPO2 heart rate, temperature with one digit fraction and display blood pressure amplitude chart over the heartbeat changing values with (the MAP, Systolic/Diastolic values, and Blood pressure heart rate). At the bottom of the screen have a text label display to give the patient some warning and troubleshoot notes.

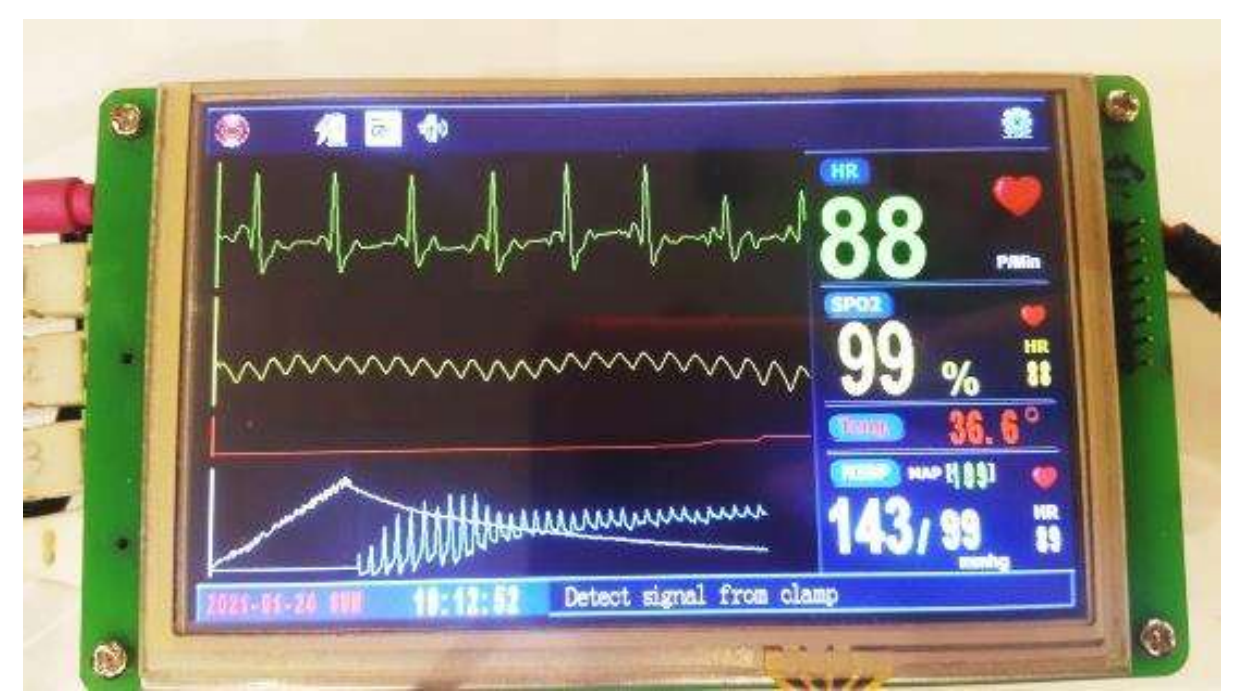

Fig. 14: The Data Displayed by Smart TFT

\subsection{Standalone ESP8266 programming}

on ESP8266 write and execute the subroutines program of the warning message and the outside range of standard medical values, to reduce the size of program flash memory in the main microcontroller program to speed up the system operation, the (see Fig. 13.b) show the flowchart of ESP8266 software, at power up the ESP8266 it will search for connecting with the last access point Wi-Fi by using the saved Network name and password after the connection is done, the BLYNK Cloud server will be Synchronized with ESP8266 by Authenticate key. the LX106 will be reading the Vital updated measuring data from the main microcontroller and sending it to the BLYNK Cloud server to displaying it on the BLYNK application of smartphone such as a simple Bridge, the LX106 will be analyses the data and comparing it with stored standard values, if found any abnormal reading result it will be sending a short note to the doctor by virtual terminal windows on a smartphone(see Fig. 15), finally, check if receive any massage from a Doctor the LX106 will be sent to the Embedded Smart TFT. 


\subsection{The Smartphone Medical programming}

The program has been designed by using BLYNK Development Application can be run under android or IOS smartphone or Tablet, the BLYNK is an IoT wireless sensing network developer program by using GUI, allow the program design to build the program by the simple and easy when starting the program it will be searching for local Wi-Fi network, the authentication Code of the BLYNK Cloud server connect the smartphone with ESP8266 which embedded in the device and synchronization the measuring data between the biomedical device and smartphone. The IoT BLYNK Development Application display same the main Measured vital processes such as ECG Heart Beat Rate, SPO2 value, body temperature value, and blood pressure Systolic and Diastolic values and write it by correct number format on the upper of the screen (see Fig. 15), the chart used to explain the changes of the vital process for many weeks to give the doctor wide seen analyses about the patient history to make a suitable diagnosis. The virtual window below the screen makes all process logs with time and gives a warning to the doctor for patient health. Also, the doctor can be sent immediately the recommendation comments or treatments in the text below to display them on the patient Smart TFT interface.

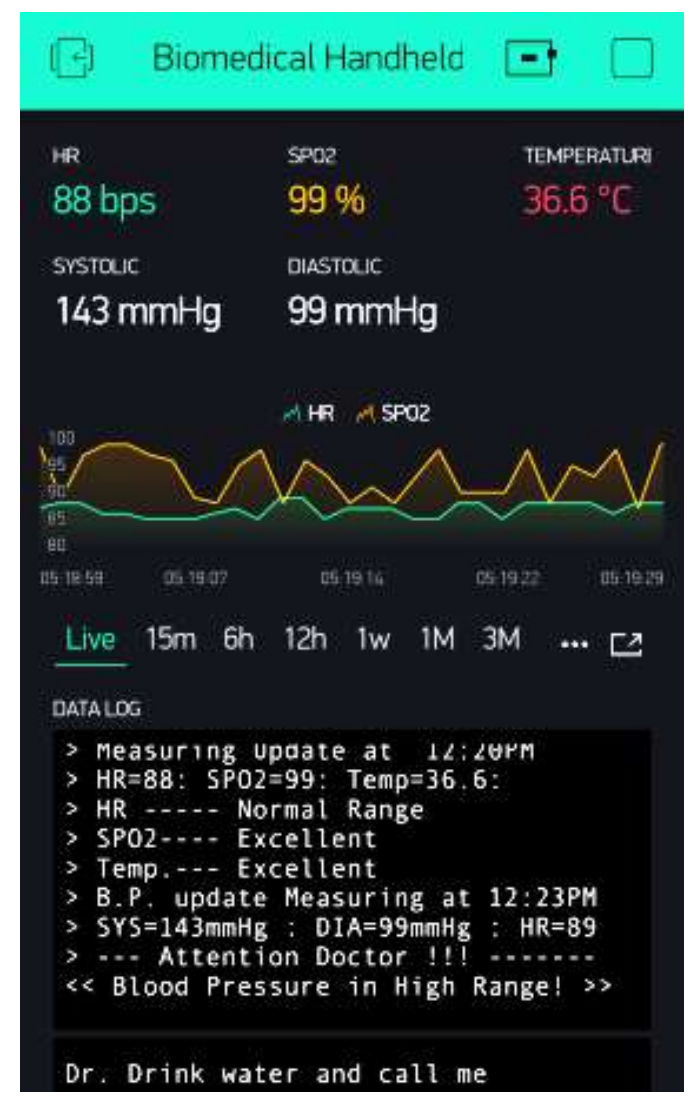

Fig. 15: The smartphone BYLNK Interface program

\subsection{Speech Alerting}

The device support a speech MP3 player, have a hazard and warning audio track some of them for troubleshooting instruction like "Check the temperature cable" or "waiting.. the Pulse Oximeter signal is calibrating", and anther warning about the patient healthy such as "low oxygen level found in the 
blood please be relaxing, take a Breath from your nose and keep it $5 \mathrm{sec}$, then push it from your mouth slowly", repeat that 10 times. all of this by many language voice support.

\section{RESULTS AND TESTING}

There are two different types of tests on the biomedical handheld prototype device; one of them is for the accuracy of the Vitals and physiological measurements of the device, and the second for testing ESP8266 with the BLYNK Cloud server services to transfer the measured vital data to the smartphone without losses.

\subsection{Test the accuracy of the vitals reading}

By comparing the results of the biomedical handheld device used in this paper results with highprecision patient Healthy monitor (MINDRAY datascope Spectrum): this device has a (UL listed and Europe CE classification). to ensure that all components work properly, all the sensors have been linked to the human body and connected to the two devices at the same time also to confirm the data communication between the side of The patient and the BLYNK Cloud server on the smartphone, and Ensure that all Vital measurements including the complete information about the patient Vital parameters, such as ECG signal, SPO2 ratio, temperature, blood pressure Systolic and Diastolic, Heartbeat signals. Check and compare the two devices to each other through 25 different patient cases, Table 2 will show only 3 different worthiest case results of the experimental tests at the ISMAILIA Medical Center, to ensure the accuracy of the system results. Record the measuring values from MINDRAY data scope and prototype 4 times every 5 minutes. "A" is (MINDRAY datascope results), "B" prototype result 
BIOMEDICAL HANDHELD EMBEDDED SYSTEM

Table 2. MINDRAY and Prototype vital sign measuring for three patients every 5 minute

\begin{tabular}{|c|c|c|c|c|c|c|c|c|c|}
\hline \multirow{2}{*}{ Parameters } & \multicolumn{8}{|c|}{ Patient (1) reading Every $5 \mathrm{~min}$} & \multirow{2}{*}{$\begin{array}{c}\text { Error } \\
\text { (\%) }\end{array}$} \\
\hline & A & B & A & B & A & B & A & B & \\
\hline ECG HR & 74 & 73 & 71 & 72 & 75 & 75 & 85 & 85 & 1.351 \\
\hline SPO2 \% & 98 & 98 & 97 & 98 & 98 & 99 & 99 & 99 & 1.031 \\
\hline SPO2 HR & 74 & 74 & 71 & 71 & 75 & 75 & 85 & 84 & 1.176 \\
\hline Temp. & 36.4 & 36.6 & 36.5 & 36.5 & 36.6 & 36.5 & 36.6 & 36.6 & 0.549 \\
\hline SBP. & 132 & 133 & 133 & 133 & 136 & 137 & 130 & 133 & 2.307 \\
\hline DBP & 83 & 82 & 87 & 84 & 90 & 89 & 88 & 89 & 3.448 \\
\hline \multirow{2}{*}{ Parameters } & \multicolumn{8}{|c|}{ Patient (2) reading Every $5 \mathrm{~min}$} & Error \\
\hline & A & B & A & B & A & B & A & B & (\%) \\
\hline ECG HR & 68 & 68 & 71 & 70 & 73 & 73 & 77 & 77 & 1.408 \\
\hline SPO2 \% & 99 & 99 & 98 & 97 & 95 & 95 & 93 & 92 & 1.075 \\
\hline SPO2 HR & 68 & 68 & 71 & 70 & 73 & 73 & 77 & 76 & 1.408 \\
\hline Temp. & 36.8 & 36.8 & 36.8 & 36.7 & 36.9 & 36.9 & 37 & 36.9 & 0.272 \\
\hline SBP. & 121 & 121 & 110 & 112 & 119 & 119 & 119 & 121 & 1.818 \\
\hline DBP & 78 & 78 & 71 & 73 & 79 & 78 & 75 & 76 & 2.817 \\
\hline \multirow{2}{*}{ Parameters } & \multicolumn{8}{|c|}{ Patient (3) reading Every $5 \mathrm{~min}$} & Error \\
\hline & A & B & A & $B$ & A & $B$ & A & B & (\%) \\
\hline ECG HR & 82 & 81 & 78 & 78 & 76 & 76 & 83 & 82 & 1.219 \\
\hline SPO2 \% & 98 & 99 & 98 & 98 & 97 & 97 & 98 & 99 & 1.020 \\
\hline SPO2 HR & 82 & 81 & 78 & 78 & 76 & 76 & 83 & 83 & 1.219 \\
\hline Temp. & 36.9 & 36.9 & 36.8 & 36.9 & 36.8 & 36.8 & 36.9 & 36.9 & 0.272 \\
\hline SBP. & 135 & 136 & 127 & 128 & 123 & 126 & 132 & 134 & 2.439 \\
\hline DBP & 86 & 86 & 85 & 84 & 84 & 83 & 86 & 88 & 2.326 \\
\hline
\end{tabular}


Table 3. Compere the errors between our prototype and 2 nearly models

\begin{tabular}{|c|c|c|c|}
\hline maximum errors & prototype & Burhanuddin et al [1] & Nastiti Virindra et al [2] \\
\hline Heart rate & $1.408 \%$ & $-0.0199-0.0021 \%$ & $2.2 \%$ \\
\hline SPO2 & $1.075 \%$ & $1.02 \%$ & - \\
\hline Temperature & $0.549 \%$ & $0.8 \%$ & Not support \\
\hline SBP & $2.439 \%$ & Not support & Not support \\
\hline DBP & $3.448 \%$ & Not support & \\
\hline
\end{tabular}

In Table 3 our prototype device is distinguished from Burhanuddin et al device[1], with higher temperature accuracy, and with Nastiti Virindra et al[2] , higher Heart rate accuracy and temperature

The ECG signal inspection tested with $A$ five points $(P, Q, R, S$, and $T$ ) with three main waves (PR interval, QRS duration [10,12], and QT interval) (see Fig. 16). In the normal adults state of the heart, the normal ECG signal range has the following:

- The PR Interval : : $120-200$ msec.

- The P wave : less than 100 msec.

- The P Volte $\quad: 0.25 \mathrm{mV}$ Height amplitude.

- The QRS duration : : $40-100$ msec.

- The QT interval : less than 400 msec. in males

less than 450 msec. in female.

- Heartbeat (PP) : from $60-100 \mathrm{bpm}$.

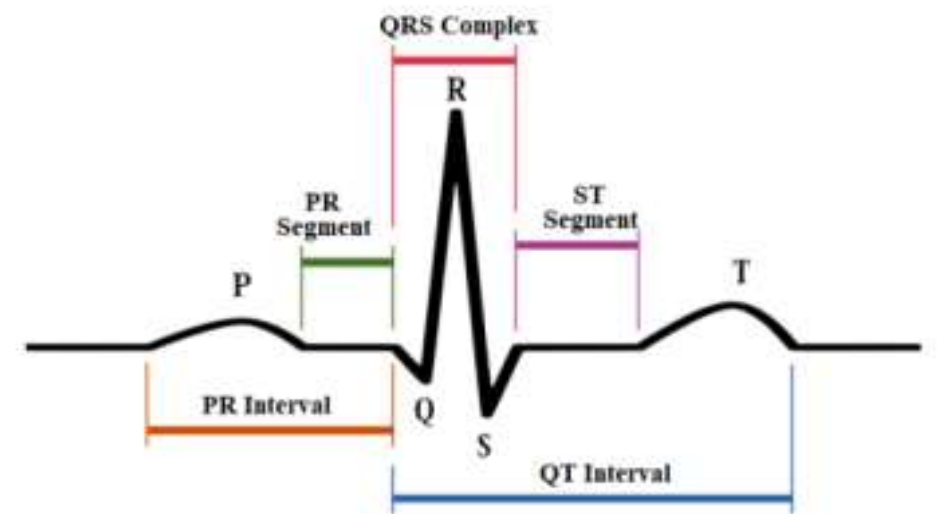

Fig. 16: ECG Wave and Interval Signal Components

Almost all results of wave shape and parameter time intervals are identical with MINDRAY recommended signal. All of them within range.

This confirmed that the proposed system is accurate in vital data, good at analyzing data, smart in decision-making, and with high reliability. 
When testing the recording of vital information on the memory SD card for more than 100 continuous hours, the system did not lose any data or stop for any reason; the $64 \mathrm{~GB}$ is fully by $19 \%$ of the total memory size

\subsection{Test the communication reliability}

Second test Reliability Test In terms of ensuring effective communication and data transmission through BLYNK the system is kept active for 100 hours continuously. And the test is whether there is any interruption during the device working, The server-side application kept the record for the time. No interruption occurred due to the hardware circuit, but there were a slowdown and delay due to the slow connection to the Internet at that time intermittent time of no more than 3 seconds, and it does not make any effect on measured data and also in this test, the prototype device confirmed that it is high communication reliability.

Table 3: compere between our device and 6 nearly model

\begin{tabular}{|c|c|c|c|c|c|c|c|}
\hline parameters & prototype & {$[1]$} & {$[2]$} & {$[3]$} & {$[4]$} & {$[5]$} & {$[11]$} \\
\hline Sensors module & $\mathbf{x}$ & $\mathbf{x}$ & $\mathbf{x}$ & $\mathbf{V}$ & $\mathbf{V}$ & $\mathbf{V}$ & $\mathbf{x}$ \\
\hline Platform or PC & $\mathbf{x}$ & $\mathbf{V}$ & $\mathbf{V}$ & $\mathbf{x}$ & $\mathbf{V}$ & $\mathbf{V}$ & $\mathbf{V}$ \\
\hline ECG Circuit & $\mathbf{V}$ & $\mathbf{V}$ & $\mathbf{V}$ & $\mathbf{V}$ & $\mathbf{x}$ & $\mathbf{x}$ & $\mathbf{V}$ \\
\hline SPO2 Circuit & $\mathbf{V}$ & $\mathbf{V}$ & $\mathbf{x}$ & $\mathbf{x}$ & $\mathbf{x}$ & $\mathbf{x}$ & $\mathbf{x}$ \\
\hline BP Circuit & $\mathbf{V}$ & $\mathbf{x}$ & $\mathbf{x}$ & $\mathbf{x}$ & $\mathbf{x}$ & $\mathbf{x}$ & $\mathbf{x}$ \\
\hline Respiration & $\mathbf{x}$ & $\mathbf{V}$ & $\mathbf{V}$ & $\mathbf{x}$ & $\mathbf{x}$ & $\mathbf{V}$ & $\mathbf{x}$ \\
\hline TFT Display & $\mathbf{V}$ & $\mathbf{x}$ & $\mathbf{V}$ & $\mathbf{x}$ & $\mathbf{V}$ & $\mathbf{x}$ & $\mathbf{V}$ \\
\hline wireless & $\mathbf{I O T}$ & $\mathbf{x}$ & $\mathbf{x}$ & $\mathbf{B T}$ & $\mathbf{x}$ & $\mathbf{I} \mathbf{T}$ & $\mathbf{x}$ \\
\hline Sound & $\mathbf{V}$ & $\mathbf{x}$ & $\mathbf{x}$ & $\mathbf{x}$ & $\mathbf{x}$ & $\mathbf{x}$ & $\mathbf{x}$ \\
\hline Store Data & $\mathbf{S D}$ & $\mathbf{x}$ & $\mathbf{x}$ & $\mathbf{V}$ & $\mathbf{x}$ & $\mathbf{V}$ & $\mathbf{V}$ \\
\hline Portable & $\mathbf{V}$ & $\mathbf{x}$ & $\mathbf{x}$ & $\mathbf{x}$ & $\mathbf{V}$ & $\mathbf{V}$ & $\mathbf{V}$ \\
\hline
\end{tabular}

\section{CONCLUSION}

Using a wide-range microcontroller, PIC18F46K22, we implemented a reliable portable medical device. The proposed device gives highly accurate measurements, compared to professional devices. Our competitive edge is the low cost without sacrificing the accuracy. The low cost is attributed to the use of commercial medical sensors and transducer available in most markets, and the Egyptian market.

We are now in the process of calibrating our device at one of the professional governmental entities to be approved for professional medical use.

The communication scheme used with our proposal is two-way, unlike most previous systems [5], where the communication is one directional; from the device to the caretaker or doctor. 
After the device gets the measurements of the vital patient's functions, the data is sent from the device to the doctor, for diagnosing and investigation. The doctor can then send the treatment or any comments via IoT to appear immediately on the patient's embedded smart TFT.

Our device is also equipped by an audio system, through voice instructions are given to the patients, to guide them connecting the sensors properly. This has proven to be very useful, especially for patients having reading impairment.

An intelligent module that makes some suggestions to help the doctor in his diagnosis also equips the device. These suggestions are sent to the doctor on his mobile phone, or his computer.

The vital processes measurements can be recorded for a period of up to 30 days without stopping, so the medical history is clear which helps getting a correct medical diagnosis.

Being low cost and easy-to-carry device (Fig. 17), makes it very suitable to reach poor areas that have little medical services.

\section{DEVELOPMENT AND FUTURE WORK}

Applying new ideas in Hardware circuit design to reduce the number of components and size of the device to obtain cost-effective and low power consumption such as common configurable signal conditioning for all sensors. for increasing the efficiency and accuracy of measurements by using an advanced DSC high range microcontroller. Add some medical invasive meters devices to measure new vital processes such as ( Blood glucose meter, urea level, carbon dioxide level .. etc), Use the upgraded type of ESP8266 such as ESP32 to make it a main host microcontroller alternative to PIC18F46K22 and also increasing the communication performance of the system.

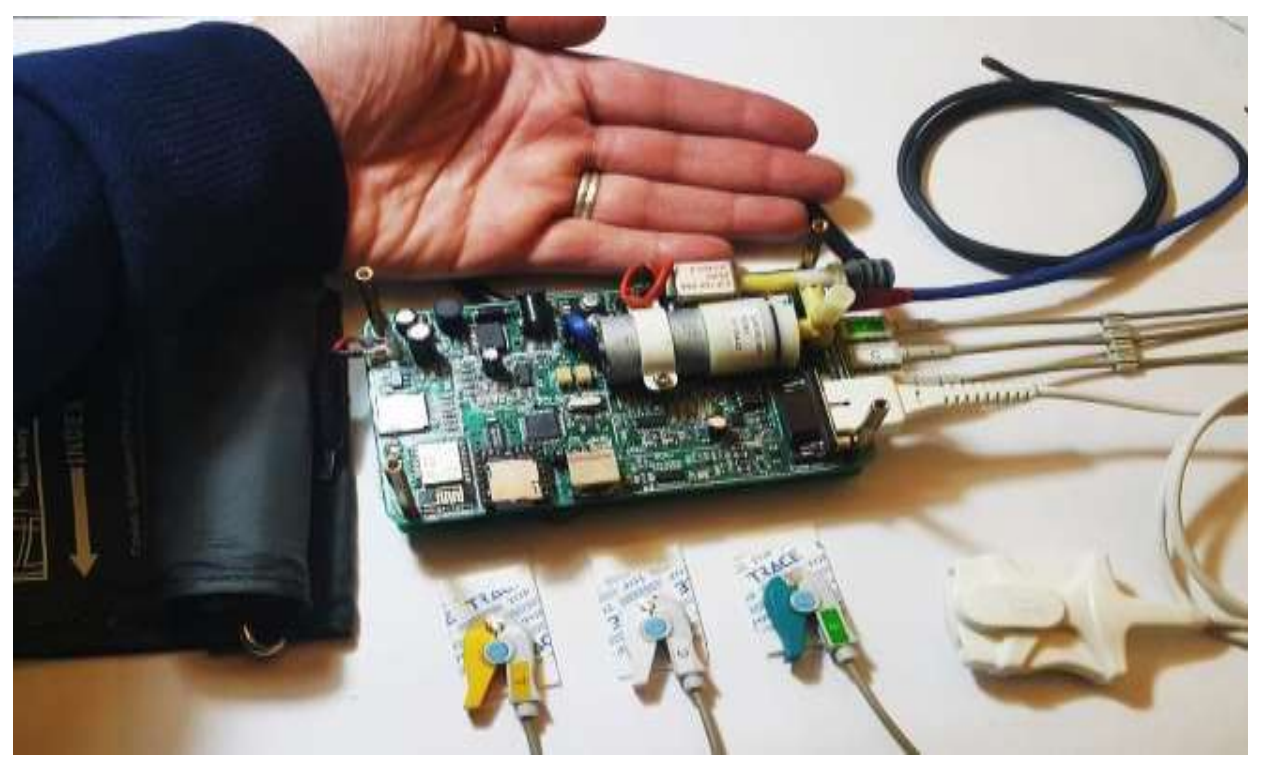

Fig. 7: Biomedical Handheld Embedded System Device

\section{REFERENCES}

1. Burhanuddin, M. A., Moch. Sahrul, T. P., Triwiyanto, T., Hamzah, T., Utomo, B., Setioningsih, E. D., \& Titisari, D. (2019) "The Design of Low-Cost Patient Monitor Based on Computer". 
International Seminar on Application for Technology of Information and Communication (iSemantic). pp. 405-410 doi:10.1109/isemantic .2019.8884346

2. Nastiti Virindra Wardhani, Ajeng Minkhata Aulia, EndroYulianto, M. Ridha Mak'ruf, Tri Bowo Indrato,(2019) "Portable Vital Sign Device with Liquid Crystal Display TFT Touchscreen" International Seminar on Application for Technology of Information and Communication (iSemantic). pp. 429-433 doi:10.1109/ISEMANTIC .2019.8884351

3. Al-Aubidy, K. M., Derbas, A. M., \& Al-Mutairi, A. W. (2016). "Real-time patient health monitoring and alarming using wireless-sensor-network". 2016 13th International MultiConference on Systems, Signals \& Devices (SSD). doi:10.1109/ ssd.2016.7473672

4. Aslam, S. K., \& Saniie, J. (2016). "Architecture and design flow of Tele-Health Monitoring system using STM32 platform". 2016 IEEE International Conference on Electro Information Technology(EIT). doi:10.1109/eit.2016.7535253

5. Zilani, K.A., Yeasmin, R., Zubair, K.A., Sammir, M.R., Sabrin, S.:"R3HMS, an IoT based approach for patient health monitoring". In: 2018 International Conference on Computer, Communication, Chemical, Material and Electronic Engineering (IC4ME2), Rajshahi, pp. 1-4 (2018). https://doi.org/10.1109/IC4ME2.2018.8465482

6. Ivan Tomašić, Roman Trobec, "Optimized Positioning of ECG Electrodes for WSN Applications", Application and Multidisciplinary Aspects of Wireless Sensor Networks Computer Communications and Networks, 2011, pp: 185-211 https:// link.springer.com/chapter/10.1007/978-1-84996-510-1_9

7. Pandey, V., \& Giri, V. K. (2016). "High frequency noise removal from ECG using moving average filters". International Conference on Emerging Trends in Electrical Electronics \& Sustainable Energy Systems, 2016 (ICETEESES). doi:10.1109/ iceteeses.2016.7581383

8. Alpert, B. S., Quinn, D., \& Gallick, D. (2014). “Oscillometric blood pressure: a review for clinicians". Journal of the American Society of Hypertension, 8(12), 930-938. doi:10.1016/j.jash.2014.08.014

9. Mittal, A., \& Rege, A. (2015). “Design of digital FIR filter implemented with window techniques for reduction of power line interference from ECG signal".2015 International Conference on Computer, Communication and Control (IC4). doi:10.1109/ic4.2015.7375535

10. G. S. Kumari, Kuswanth Kumar, J. Anusha, \& M.P. Rao, "Electrocardiographic signal analysis using wavelet transforms", Intr. Conf. on Electrical Electronics Signals Communication and Optimization (EESCO), 2015, pp:1-6. doi:10.1109/SSD .2016.7473672

11. Jeon, T., Kim, B., Jeon, M., \& Lee, B.-G. (2014).” Implementation of a portable device for realtime ECG signal analysis". BioMedical Engineering OnLine, 13(1), 160. doi:10.1186/1475$925 \mathrm{x}-13-160$.

12. S. Gradl, P. Kugler, C. Lohmuller, \& B. Eskofier, "Real-time ECG monitoring and arrhythmia detection using Android-based mobile devices", Annual IEEE Intr. Conf. on Engineering in Medicine and Biology Society, 2012, pp:2452-2455. doi:10.1109/embc.2014.6944177

13. World Health Organization, https://www.who.int/news-room/fact-sheets/detail/ cardiovasculardiseases-(cvds), 2016

14. 8-bit PIC Microcontroller peripherals integration, https://www.microchip.com/content/ $\mathrm{dam} / \mathrm{mchp} /$ documents/OTH/ProductDocuments/Brochures/30010068G.pdf

15. Pulse Oximeter Design Using Microchip's Analog Devices and dsPIC $®$ Digital Signal Controllers (DSCs) https://www.microchip.com/content/dam/mchp/documents/ OTH/ApplicationNotes/ApplicationNotes/00001525B.pdf

16. http://www.patarnott.com/atms360/Arduino/pdf/negativeTemperatureCoefThermistors.pdf , NTC Thermistors Engineering Notes 
17. https://www.ti.com/lit/an/sloa024b/sloa024b.pdf?ts=1612093230014\&ref_url=https\%253A\%2 52F\%252Fwww.google.com\%252F , Analysis of Sallen Key Architecture.

18. http://ww1.microchip.com/downloads/en/AppNotes/00001556B.pdf . Blood Pressure Meter Design Using Microchip's Analog Devices and PIC24F Microcontrollers, 2015

19. http://ww1.microchip.com/downloads/en/AppNotes/00001525B.pdf, Pulse Oximeter Design Using Microchip's Analog Devices and dsPIC® Digital Signal Controllers (DSCs) , 2015

20. https://www.espressif.com/sites/default/files/documentation/0aesp8266ex datasheet en.pdf. ESP8266 Datasheet, version 6.6, 2020

21. http://dwin.com.cn/home/English/lcm_info. DUGS, graphics application service software, website

22. https://www.espressif.com/sites/default/files/documentation/5a esp8266_sdk_ssl_user_manual_en.pdf 